\title{
EHMTI-0252. The frequency of acute medication intake relates to its perceived effectiveness in chronic headache patients
}

\author{
DML Van Ryckeghem ${ }^{1 *}$, T Wens ${ }^{1}$, K Paemeleire $^{2}$, G Crombez $^{1}$ \\ From 4th European Headache and Migraine Trust International Congress: EHMTIC 2014 \\ Copenhagen, Denmark. 18-21 September 2014
}

\section{Introduction}

Although the benefit of acute pain medications for chronic headaches ( $\geq 15$ days/month) often is limited, patients frequently continue to take them in an attempt to control their pain, which may then result in medication overuse.

\section{Aims}

The current study investigates whether the intake of acute medication creates an illusion of control (IoC) and whether an accepting attitude may prevent this IoC in chronic headache patients.

\section{Method}

Forty-one chronic headache patients (primarily migraine sufferers) filled out a questionnaire battery (e.g. CPAQ and MIDAS) followed by an adapted IoC Task. During this IoC task, patients were asked on each trial to indicate whether they would (fictively) take a new painkiller to cope with a (fictive) headache day. Afterwards the patient received random information on the effectiveness of the painkiller that day. After the task participants filled out 6 questions on the perceived effectiveness of this painkiller.

\section{Results}

Data show that the perceived effectiveness was positively related to the number of times patients have fictively taken the painkiller $(\mathrm{p}<.01)$ and not to its actual contingency (i.e., actual effectiveness). Secondly, acceptance was positively related to perceived effectiveness of the painkiller $(\mathrm{p}<.05)$, but did not moderate the relationship between perceived effectiveness and frequency of medication intake.

\section{Conclusions}

Results of the present study suggest that a higher intake of acute medication by chronic headache patients is related to higher perceived effectiveness of the acute medication, independent of its actual effectiveness. This IoC-effect is however not influenced by acceptance.

No conflict of interest.

Authors' details

${ }^{1}$ Department of Experimental Clinical and Health Psychology, Ghent University, Ghent, Belgium. ${ }^{2}$ Department of Neurology, University Hospital Ghent, Ghent, Belgium.

Published: 18 September 2014

doi:10.1186/1129-2377-15-S1-E23

Cite this article as: Van Ryckeghem et al:: EHMTI-0252. The frequency of acute medication intake relates to its perceived effectiveness in chronic headache patients. The Journal of Headache and Pain 201415 (Suppl 1):E23 